\title{
A NOTE ON EXISTENCE OF ENVELOPES AND COVERS
}

\author{
JiAnlong Chen and Nanging Ding
}

\begin{abstract}
We prove the following results for a ring $R$. (a) If $\mathcal{C}$ is a class of right $R$-modules closed under direct summands and isomorphisms, then every right $R$-module has an epic $\mathcal{C}$-envelope if and only if $\mathcal{C}$ is closed under direct products and submodules. (b) If $R$ is left $\tau$-coherent and pure injective as a right $R$-module, then every $\mathcal{T}$-finitely presented right $R$-module has a $\mathcal{T}$-flat envelope. (c) Let $R$ be a left $\mathcal{T}$ coherent ring and injective right $R$-modules be $\mathcal{T}$-flat. If every finitely presented left $R$-module has a flat envelope, then every $\mathcal{T}$-finitely presented right $R$-module has a projective cover.
\end{abstract}

\section{INTRODUCTION}

Throughout this paper, $R$ will denote an associative ring with identity and all modules will be unitary.

If $R$ is a ring and $\mathcal{C}$ a subclass of the category $\operatorname{Mod}_{R}$ of right $R$-modules, by a $\mathcal{C}$ pre-envelope of a right $R$-module $M$ we mean a homomorphism $\phi: M \rightarrow F$ with $F \in \mathcal{C}$ such that for any homomorphism $f: M \rightarrow F^{\prime}$ where $F^{\prime} \in \mathcal{C}$ there is a homomorphism $g: F \rightarrow F^{\prime}$ such that $g \phi=f$. If, furthermore, when $F^{\prime}=F$ and $f=\phi$, the only such $g$ are automorphisms of $F$, then $\phi$ is called a $\mathcal{C}$-envelope of $M$. If $\mathcal{C}$ is the class of injective modules, then we get the usual injective envelopes. For $\mathcal{C}$ some familiar class of modules, say the class of flat (respectively finitely projective, projective) modules, $\mathcal{C}$-envelopes will simply be called flat (respectively finitely projective, projective) envelopes. If envelopes exist they are unique up to isomorphism. $\mathcal{C}$-(pre)covers can be defined dually.

The question on the existence of envelopes and covers has been studied by many authors (see for example, $[1,2,6,7,9,11]$ ). In this paper, we first show that if $\mathcal{C}$ is a class of right $R$-modules closed under direct summands and isomorphisms, then every right $R$-module has an epic $\mathcal{C}$-envelope if and only if $\mathcal{C}$ is closed under direct products and submodules (Theorem 2). It is not known over which rings every module has an $F P$-injective cover. But, as an immediate consequence of the dual of Theorem 2, we

Received 7 December 1995

This work was carried out during a visit of the first author to the Academia Sinica, China and a visit of the second author to the University of Tübingen, Germany. The authors would like to thank the Institutes of Mathematics in both institutions for their kind hospitality.

Copyright Clearance Centre, Inc. Serial-fee code: 0004-9729/96 \$A2.00+0.00. 
have that a ring $R$ is right semihereditary if and only if every right $R$-module has a monic $F P$-injective cover. Then, by a result of Gruson and Jensen [12], it is shown that if $R$ is left $\mathcal{T}$-coherent and pure injective as a right $R$-module, then every $\mathcal{T}$-finitely presented right $R$-module has a $\mathcal{T}$-flat envelope (Theorem 10). Finally, let $R$ be a left $\mathcal{T}$-coherent ring and let injective right $R$-modules be $\mathcal{T}$-flat, we prove that if every finitely presented left $R$-module has a flat envelope, then every $\mathcal{T}$-finitely presented right $R$-module has a projective cover (Theorem 14). In particular, some known results are obtained as corollaries of the main results of this paper.

\section{Preliminaries}

In this section we recall some known notions and facts which we need in the later section.

(1) Finite (local) projectivity. An $R$-module $M$ is called finitely (respectively locally) projective $[3,14]$ if, for any finitely generated submodule $M_{0}$ of $M$, there exist a finitely generated free module $F$ and homomorphisms $f: M_{0} \rightarrow F$ (respectively $f: M \rightarrow F)$ and $g: F \rightarrow M$ such that $g(f(x))=x$ for all $x \in M_{0}$. Finitely projective modules were called $f$-projective in [14]. In general, projective $\Rightarrow$ locally projective $\Rightarrow$ finitely projective $\Rightarrow$ flat, but no two of these concepts are equivalent.

(2) Relative flatness. In [6], we defined the concept of flat modules with respect to an arbitrary torsion theory. Here we recall this definition in a more general setting. Let $\mathcal{T}$ be a subclass of $\operatorname{Mod}_{R}$ with $0 \in \mathcal{T}$. A right $R$-module $M$ is said to be $\mathcal{T}$ finitely generated if $M / M^{\prime} \in \mathcal{T}$ for some finitely generated submodule $M^{\prime}$ of $M$. $M$ is said to be $\mathcal{T}$-finitely presented if there is an exact sequence $0 \rightarrow K \rightarrow F \rightarrow M \rightarrow 0$ with $F$ finitely generated free and $K \mathcal{T}$-finitely generated. $M$ is called $\mathcal{T}$-flat if every homomorphism from a $\mathcal{T}$-finitely presented $R$-module to $M$ can be factored through a finitely generated free module, that is, for any $\mathcal{T}$-finitely presented $R$-module $P$ and any homomorphism $f: P \rightarrow M$, there exist a finitely generated free module $F$ and homomorphisms $g: P \rightarrow F$ and $h: F \rightarrow M$ such that $f=h g$. It is clear that every finitely projective module is $\mathcal{T}$-flat, and every $\mathcal{T}$-flat module is flat. If $\mathcal{T}=\{0\}\left(\right.$ respectively $\left.\operatorname{Mod}_{R}\right)$, then an $R$-module $M$ is $\mathcal{T}$-flat if and only if $M$ is flat (respectively finitely projective).

(3) Coherent rings. A ring $R$ is said to be left coherent if every finitely generated left ideal of $R$ is finitely presented, or equivalently, any direct product of copies of $R$ is a flat right $R$-module. $R$ is called left $\Pi$-coherent $[4,14]$ if every finitely generated torsionless left $R$-module is finitely presented, or equivalently, any direct product of copies of $R$ is a finitely projective right $R$-module [14]. $R$ is called strongly left coherent [18] if any direct product of copies of $R$ is a locally projective right $R$-module. Note that $\Pi$-coherent rings were called strongly coherent rings by Jones [14]. $R$ is called left 
$\mathcal{T}$-coherent [6] if any direct product of copies of $R$ is a $\mathcal{T}$-flat right $R$-module. Clearly, if $\mathcal{T}=\{0\}$ (respectively $\operatorname{Mod}_{R}$ ), then $R$ is left coherent (respectively $\Pi$-coherent).

\section{Main Results}

We start with

LEMMA 1 . Let $\mathcal{C}$ be a class of right $R$-modules closed under direct summands. If every right $R$-module has a $\mathcal{C}$-pre-envelope, then $\mathcal{C}$ is closed under direct products.

Proof: For any family $\left\{F_{i}\right\}_{i \in I} \subseteq \mathcal{C}, \prod F_{i}$ has a $\mathcal{C}$-pre-envelope $\phi: \prod F_{i} \rightarrow F$ by hypothesis. Let $p_{i}: \prod F_{i} \rightarrow F_{i}$ be the projection. Then there exists $\psi_{i}: F \rightarrow F_{i}$ such that $\psi_{i} \phi=p_{i}, i \in I$. Define $\psi: F \rightarrow \prod F_{i}$ by $\psi(x)=\left(\psi_{i}(x)\right)$ for $x \in F$. For any $\left(x_{i}\right) \in \prod F_{i}$, let $\phi\left(\left(x_{i}\right)\right)=x$, then

$$
x_{i}=p_{i}\left(\left(x_{i}\right)\right)=\psi_{i} \phi\left(\left(x_{i}\right)\right)=\psi_{i}(x)
$$

and hence

$$
\psi \phi\left(\left(x_{i}\right)\right)=\psi(x)=\left(\psi_{i}(x)\right)=\left(x_{i}\right)
$$

that is, $\psi \phi=1$. Thus $\prod F_{i}$ is a direct summand of $F$, and so $\prod F_{i} \in \mathcal{C}$ by assumption.

TheOREM 2. Let $\mathcal{C}$ be a class of right $R$-modules closed under direct summands and isomorphisms. Then the following are equivalent.

(1) Every right $R$-module has an epic $\mathcal{C}$-envelope.

(2) $\mathcal{C}$ is closed under direct products and submodules.

Proof: $(1) \Rightarrow(2)$. $\mathcal{C}$ is closed under direct products by Lemma 1 . For any submodule $K$ of a right $R$-module $N \in \mathcal{C}$, since $K$ has an epic $\mathcal{C}$-envelope $f: K \rightarrow F$, there is a homomorphism $g: F \rightarrow N$ such that $g f=i$, where $i: K \rightarrow N$ is the inclusion map. Thus $f$ is monic, and so $K \cong F \in \mathcal{C}$.

$(2) \Rightarrow(1)$. Let $X$ be any right $R$-module and let $\left\{N_{i}\right\}_{i \in I}$ be the family of all the submodules of $X$ such that $X / N_{i} \in \mathcal{C}$. Let $\mathcal{C}(X)=X / \bigcap_{i \in I} N_{i}$ and $\pi: X \rightarrow \mathcal{C}(X)$ be the quotient map. Define $\lambda: \mathcal{C}(X) \rightarrow \prod_{i \in I} X / N_{i}$ via $\lambda\left(x+\bigcap_{i \in I} N_{i}\right)=\left(x+N_{i}\right)$ for $x \in X$. Then $\lambda$ is a monomorphism. Since $X / N_{i} \in \mathcal{C}, \prod_{i \in I} X / N_{i} \in \mathcal{C}$. So $\mathcal{C}(X) \in \mathcal{C}$. For any $F \in \mathcal{C}$ and any $\phi: X \rightarrow F, X / \operatorname{Ker}(\phi) \in \mathcal{C}$ since $X / \operatorname{Ker}(\phi) \cong \operatorname{Im}(\phi) \subseteq F$, and hence $\operatorname{Ker}(\phi)=N_{\alpha}$ for some $\alpha \in I$. Now define $\xi: \mathcal{C}(X) \rightarrow F$ such that $\xi\left(x+\bigcap_{i \in I} N_{i}\right)=\phi(x)$ for $x \in X$. Then $\xi$ is well-defined (for $\operatorname{Ker}(\phi)=N_{\alpha}$ ), and $\xi \pi=\phi$. This shows that $\pi$ is a $\mathcal{C}$-pre-envelope. Since $\pi$ is epic, $\pi$ is a $\mathcal{C}$-envelope. This completes the proof. 
As applications, we list some corollaries of the Theorem 2 above.

Let $\mathcal{C}$ be the class of $\mathcal{T}$-flat right $R$-modules. Clearly, $\mathcal{C}$ is closed under isomorphisms and direct summands. By [6, Proposition 3.4], $\mathcal{C}$ is closed under direct products if and only if $R$ is left $\mathcal{T}$-coherent. Thus we have

Corollary 3. ([6, Theorem 5.1].) The following are equivalent for a ring $R$.

(1) $R$ is left $\mathcal{T}$-coherent and every submodule of a $\mathcal{T}$-flat right $R$-module is T-flat.

(2) Every right $R$-module has an epic $\mathcal{T}$-flat envelope.

Recall that a ring $R$ is strongly left coherent if and only if any direct product of locally projective right $R$-modules is locally projective [18]. So we obtain

Corollary 4. ([6, Proposition 5.4].) The following are equivalent for a ring $R$.

(1) $R$ is strongly left coherent and submodules of locally projective right $R$-modules are locally projective.

(2) Every right $R$-module has an epic locally projective envelope.

It is well known that $R$ is left coherent and right perfect if and only if every direct product of projective right $R$-modules is projective. Therefore we get

Corollary 5. ([6, Proposition 5.5].) The following are equivalent for any ring $R$.

(1) $R$ is left semihereditary and right perfect.

(2) $R$ is left coherent, right perfect and right hereditary.

(3) Every right $R$-module has an epic projective envelope.

Proof: $(1) \Leftrightarrow(2)$ is easy.

(2) $\Leftrightarrow$ (3) by Theorem 2 .

Now we state the dual of Theorem 2.

Theorem 6. ([11, Proposition 4].) Let $\mathcal{C}$ be a class of right $R$-modules closed under direct summands and isomorphisms. Then the following are equivalent.

(1) Every right $R$-module has a monic $\mathcal{C}$-cover.

(2) $\mathcal{C}$ is closed under direct sums and homomorphic images.

Let $\mathcal{C}$ be the class of injective right $R$-modules in Theorem 6 . We obtain

COROLLARY 7. The following are equivalent for any ring $R$.

(1) Every right $R$-module has a monic injective cover.

(2) $R$ is right Noetherian and right hereditary. 
REMARK 1. We note that Corollary 7 above appeared in [8, Corollary 3.4].

Recall that an $R$-module $M$ is called $F P$-injective (or absolutely pure) $[15,16]$ if $\operatorname{Ext}_{R}^{1}(N, M)=0$ for all finitely presented $R$-modules $N$. It is well known that the class of $F P$-injective modules over any ring is closed under direct sums [15, Corollary], and a ring $R$ is right semihereditary if and only if the class of $F P$-injective right $R$ modules is closed under homomorphic images [16, Theorem 2]. Thus, if $\mathcal{C}$ is the class of $F P$-injective modules in Theorem 6, one gets the following corollary which characterises semihereditary rings in terms of $F P$-injective covers.

Corollary 8. The following are equivalent for any ring $R$.

(1) Every right $R$-module has a monic $F P$-injective cover.

(2) $R$ is right semihereditary.

In order to prove the next main result, we need a result of Gruson and Jensen [12]. In the notation of [12], if $P f(R)$ denotes the full subcategory of the category of left $R$-modules whose objects are the finitely presented left $R$-modules and $D(R)$ is the Grothendieck category of additive functors from $P f(R)$ to Abelian groups, we have the following characterisation of the injective objects in this category.

Lemma 9 . ([12, Proposition 1.2].) An object $F$ of $D(R)$ is injective if and only if $F$ is naturally equivalent to a functor $E \otimes-$ with $E$ a right pure injective module.

Theorem 10. Let $R$ be left $\mathcal{T}$-coherent and pure injective as a right $R$-module. Then every $\mathcal{T}$-finitely presented right $R$-module has a $\mathcal{T}$-flat (or projective) envelope.

Proof: Let $M$ be a $\mathcal{T}$-finitely presented right $R$-module, then $M$ has a $\mathcal{T}$-flat pre-envelope $\phi: M \rightarrow F^{\prime}$ by [6, Theorem 3.10]. In view of the $\mathcal{T}$-flatness of $F^{\prime}$, there exist a finitely generated free right $R$-module $F$ and homomorphisms $f: M \rightarrow F$ and $f^{\prime}: F \rightarrow F^{\prime}$ such that $\phi=f^{\prime} f$. It is easy to see that $f: M \rightarrow F$ is a $\mathcal{T}$-flat pre-envelope of $M$. Since $R$ is pure injective as a right $R$-module, $F$ is a right pure injective module, and hence $F \otimes-$ is an injective object of $D(R)$ by Lemma 9 . To prove that $M$ has a $T$-flat envelope, we use an argument similar to that in [2, Theorem 3.3]. If $E \otimes-$ is the injective hull of the image functor $G=\operatorname{Im}(f \otimes-)$, then $E \cong E \otimes R$ is a direct summand of $F \cong F \otimes R$ and so it is finitely generated projective (obviously, it is $\mathcal{T}$-flat). Moreover, $f$ factors through $M \rightarrow G(R) \rightarrow E$, from which we obtain that if $g$ is the above composition, it is a $\mathcal{T}$-flat pre-envelope of $M$. Now, each endomorphism $h$ of $E$ such that $h g=g$ induces an endomorphism $h \otimes-$ of $E \otimes-$ in $D(R)$, whose restriction to $G$ is the canonical inclusion of $G$ in its injective hull and so $h \otimes-$ is an isomorphism in $D(R)$. In particular, $h$ is an isomorphism, which proves that $g: M \rightarrow E$ is a $\mathcal{T}$-flat envelope of $M$. Since $E$ is projective, $g: M \rightarrow E$ is also a projective envelope.

Since injective modules are always pure injective, we have 
COROLlary 11. If $R$ is left $\mathcal{T}$-coherent and right self-injective, then every $\mathcal{T}$ finitely presented right $R$-module has a $T$-flat (or projective) envelope.

By specialising Theorem 10 to the case $\mathcal{T}=\{0\}$, we obtain the following result of [9] immediately as a corollary.

Corollary 12. ([9, Corollary 2.4].) If $R$ is left coherent and pure injective as a right $R$-module, then every finitely presented right $R$-module has a flat (or projective) envelope.

Let $\mathcal{T}=\operatorname{Mod}_{R}$ in Theorem 10. One gets

Corollary 13. If $R$ is left II-coherent and pure injective as a right $R$-module, then every finitely generated right $R$-module has a (finitely) projective envelope.

Next we consider when every $\mathcal{T}$-finitely presented right $R$-module has a projective cover.

ThEOREM 14. Let $R$ be a left $\mathcal{T}$-coherent ring and let injective right $R$-modules be $\mathcal{T}$-flat. If every finitely presented left $R$-module has a flat envelope, then every $T$ finitely presented right $R$-module has a projective cover.

Proof: Let $M$ be a $\mathcal{T}$-finitely presented right $R$-module, then $M^{*}$ is finitely presented by [6, Proposition 3.4] since $R$ is left $\mathcal{T}$-coherent. From the hypothesis that every finitely presented left $R$-module has a flat envelope we know that $R$ is right coherent by [1, Proposition 2] and $M^{*}$ has a flat envelope, and so $M^{* *}$ has a projective cover by [1, Proposition 1]. Next we shall show that $M$ is reflexive, and hence $M$ has a projective cover. In fact, let $F \rightarrow M \rightarrow 0$ be exact with $F$ finitely generated free. Then we get an exact sequence $0 \rightarrow M^{*} \rightarrow F^{*} \rightarrow N \rightarrow 0$, where $N=F^{*} / M^{*}$. Since $M^{*}$ is finitely generated, $N$ is a finitely presented left $R$-module. By assumption, every injective right $R$-module is flat, and so $R$ is left $F P$-injective by [13, Theorem 3.3]. Therefore we obtain the following commutative diagram with exact rows:

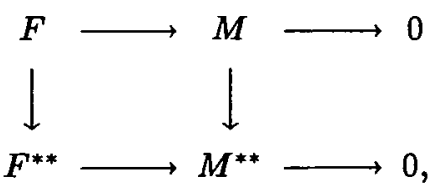

and so $M \rightarrow M^{* *}$ is an epimorphism. On the other hand, since the injective envelope $E(M)$ of $M$ is $\mathcal{T}$-flat, the inclusion map $i: M \rightarrow E(M)$ can be factored through a finitely generated free module. Thus $M$ can be embedded in a free module, and so $M$ is torsionless, that is, $M \rightarrow M^{* *}$ is a monomorphism. Consequently $M$ is reflexive. The proof is complete.

We recall that $R$ is said to be semiregular [17] if each finitely presented right (or left) $R$-module has a projective cover. $R$ is called right $I F[13]$ if every injective right $R$-module is flat. Let $\mathcal{T}=\{0\}$ in Theorem 14. We have 
COROLlaRY 15. Let $R$ be left coherent and right IF. If every finitely presented left $R$-module has a flat envelope, then $R$ is semiregular.

COROLlary 16. If $R$ is a commutative $I F$ ring, then $R$ is semiregular if and only if every finitely presented $R$-module has a flat envelope.

Proof: Since $R$ is commutative $I F, R$ is coherent by [5, Corollary 3.14]. Thus the necessity is clear by [1, Corollary 3], and the sufficiency follows from Corollary 15 .

REMARK 2. We note that the Corollary 16 above was obtained in [1] where the authors gave an $I F$ ring without sufficient flat envelopes, even for finitely presented modules (see $[1, \mathrm{p} .125-126])$.

Recall that a ring $R$ is semiperfect if every finitely generated right (or left) $R$ module has a projective cover. $R$ is called right $F G F[10]$ if every finitely generated right $R$-module embeds in a free right $R$-module. $R$ is right $F G F$ if and only if every injective right $R$-module is finitely projective [14, Theorem 2.10]. If $\mathcal{T}=\operatorname{Mod}_{R}$ in Theorem 14, we obtain

COROLlary 17. Let $R$ be left II-coherent and right FGF. If every finitely presented left $R$-module has a flat envelope, then $R$ is semiperfect.

\section{REFERENCES}

[1] J. Asensio Mayor and J. Martinez Hernandez, 'Flat envelopes in commutative rings', Israel J. Math. 62 (1988), 123-128.

[2] J. Asensio Mayor and J. Martinez Hernandez, 'On flat and projective envelopes', J. Algebra 160 (1993), 434-440.

[3] G. Azumaya, 'Finite splitness and finite projectivity', J. Algebra 106 (1987), 114-134.

[4] V. Camillo, 'Coherence for polynomial rings', J. Algebra 132 (1990), 72-76.

[5] N.Q. Ding and J.L. Chen, 'The flat dimensions of injective modules', Manuscripta Math. 78 (1993), 165-177.

[6] N.Q. Ding and J.L. Chen, 'Relative coherence and preenvelopes', Manuscripta Math. 81 (1993), 243-262.

[7] E.E. Enochs, 'Injective and flat covers, envelopes and resolvents', Israel J. Math. 39 (1981), 189-209.

[8] E.E. Enochs and O. Jenda, 'Resolvents and dimensions of modules and rings', Arch. Math. 56 (1991), 528-532.

[9] E.E. Enochs, O. Jenda and J. Xu, 'The existence of envelopes', Rend. Sem. Mat. Univ. Padova 90 (1993), 45-51.

[10] C. Faith, 'Embeding torsionless modules in projectives', Publ. Mat. 34 (1990), 379-387.

[11] J.R. García Rozas and B. Torrecillas, 'Relative injective covers', Comm. Algebra 22 (1994), 2925-2940. 
[12] L. Gruson and C.U. Jensen, 'Dimensions cohomologiques relieés aux foncteurs $\lim ^{(i)}$, , in Séminaire d'Algèbre Paul Dubreil et Marie-Paule Malliavin, Lecture Notes in Math. 867 (Springer-Verlag, Berlin, Heidelberg, New York, 1981), pp. 234-294.

[13] S. Jain, 'Flat and FP-injectivity', Proc. Amer. Math. Soc. 41 (1973), 437-442.

[14] M.F. Jones, 'Flatness and $f$-projectivity of torsion free modules and injective modules', in Advances in Non-Commutative Ring Theory, Lecture Notes in Math. 951 (Springer-Verlag, Berlin, Heidelberg, New York, 1982), pp. 94-116.

[15] B. Maddox, 'Absolutely pure modules', Prac. Amer. Math. Soc. 18 (1967), 155-158.

[16] C. Megibben, 'Absolutely pure modules', Proc. Amer. Math. Soc. 26 (1970), 561-566.

[17] W.K. Nicholson, 'Semiregular modules and rings', Canad. J. Math. 28 (1976), 1105-1120.

[18] B. Zimmermann-Huisgen, 'Pure submodules of direct products of free modules', Math. Ann. 224 (1976), 233-245.

Department of Mathematics and Mechanics

Southeast University

Nanjing 210096

People's Republic of China
Department of Mathematics

Nanjing University

Nanjing 210093

People's Republic of China 